\title{
Perception of computerised predictions using cephalometric software facad in orthognathic surgery
}

\author{
Abhimanyu Rohmetra ${ }^{1 *}$, Niharika Gupta ${ }^{2}$, Ankita Jaiswal ${ }^{3}$, Abhimanyu Vikram Singh ${ }^{4}$, \\ Rachana Singh ${ }^{5}$ \\ ${ }^{1,3,5}$ Senior Lecturer, ${ }^{2}$ Post Graduate Student, ${ }^{4}$ Consultant,,${ }^{1,3,4}$ Dept. of Orthodontics, ${ }^{2}$ Dept. of Oral Surgery, \\ ${ }^{1}$ Institute of Dental Sciences, Sehora, Jammu, Jammu \& Kashmir, India, ${ }^{2}$ D.A.V Centenary Dental College, Yamuna Nagar, \\ Haryana, India, ${ }^{3}$ Vyas Dental College and Hospital, Jodhpur, Rajasthan, India, ${ }^{4}$ Shri Harbhaj Ram Kripa Devi Trust Hospital, \\ Charbagh, Lucknow, Uttar Pradesh, India, ${ }^{5}$ Career Dental College, Lucknow, Uttar Pradesh, India \\ *Corresponding Author: Abhimanyu Rohmetra \\ Email: dr.ortho.aj@gmail.com
}

\begin{abstract}
Introduction: Orthognathic surgery has become a more acceptable treatment modality in conjunction with orthodontic treatment as esthetic awareness has been increased. The criteria for success of an orthognathic surgical procedure are not only centred on the correction of the skeletal and dental abnormalities, but also on an esthetic improvement as judged by both patients and practitioners. Using computer softwares for prediction of orthognathic surgical outcome is gaining more popularity. It was the purpose of the present study to examine the efficacy of prediction of soft tissue changes after performing orthognathic surgeries using the cephalometric software program FACAD.

Aims and Objectives: The aim of the study is to assess the perception of facial profile attractiveness and soft tissue outcome in predicted profile images in patients undergoing orthognathic surgery.

Materials and Methods: Total of 50 samples aged 20 to 25 years (35 females and 15 males) 20 skeletal class I bimaxillary protrusion cases, 20 skeletal class II cases and 10 skeletal class III cases. Cephalometric radiographs were scanned using Epson V 700 film scanner. The radiographic images were analysed using cephalometric software (FACAD 3.6 )and traced by identifying and placing a series of cephalometric points, both bony and soft tissue.

Result: The study suggested that the predicted profile images of all the patients were attractive and rankings were decreasing from class I bimaxillary class III and then class II. The least score of attractiveness were given to class II patients. The predicted profile images of class I bimaxillary patients were more attractive.

Conclusion: this study showed that the predicted profile images were attractive as suggested by three raters with class I bimaxillary most attractive and class II least attractive. In overall, laypersons and dental students ratings were somehow similar in the assessment of facial profile attractiveness in the predicted profile images.
\end{abstract}

Keywords: Orthognathic surgery, FACAD, Attractiveness

\section{Introduction}

Orthognathic surgery has become a more acceptable treatment modality in conjunction with orthodontic treatment as esthetic awareness has been increased ${ }^{1}$. The criteria for success of an orthognathic surgical procedure are not only centred on the correction of the skeletal and dental abnormalities, but also on an esthetic improvement as judged by both patients and practitioners. ${ }^{2}$ Using computer softwares for prediction of orthognathic surgical outcome is gaining more popularity. Schendel, Eisenfeld, Bell and Epker were among the first to employ a computer system for the analysis of preoperative and post operative soft tissue profile. It was the purpose of the present study to examine the efficacy of prediction of soft tissue changes after performing orthognathic surgeries using the cephalometric software program FACAD

\section{Aims and Objectives}

The aim of the study is to assess the perception of facial profile attractiveness and soft tissue outcome in predicted profile images in patients undergoing orthognathic surgery. The objective of the study is to predict how efficient is the cephalometric software (FACAD) in predicting the soft tissue profile outline in patients undergoing orthognathic surgery.

\section{Materials and Methods}

Total of 50 samples aged 20 to 25 years ( 35 females and 15 males) 20 skeletal class I bimaxillary protrusion cases, 20 skeletal class II cases and 10 skeletal class III cases.

\section{Method}

Cephalometric radiographs were scanned using Epson V 700 film scanner. The radiographic images were analysed using cephalometric software (FACAD 3.6) 
and traced by identifying and placing a series of cephalometric points, both bony and soft tissue.Then soft tissue profiles are outlined. Cephalograms are matched to the profile digital images. Then planning for orthognathic surgery is done based on the cephalometric analysis.

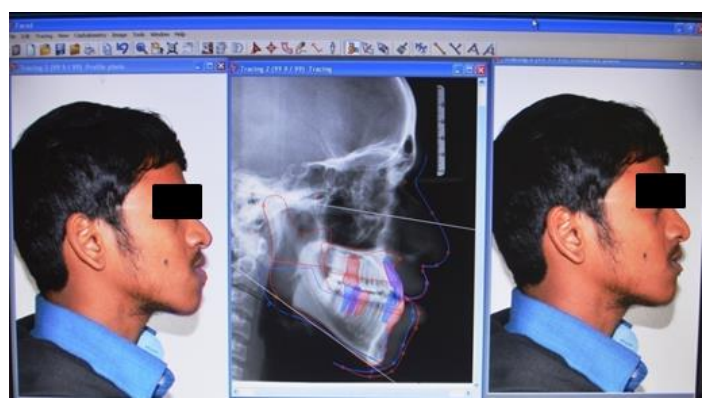

Fig. 1: Working on cephalometric software FACAD 3.6

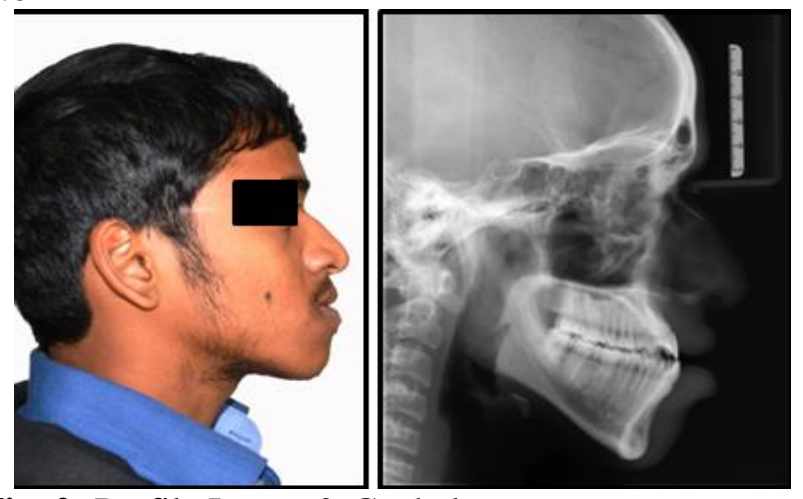

Fig. 2: Profile Image \& Cephalogram

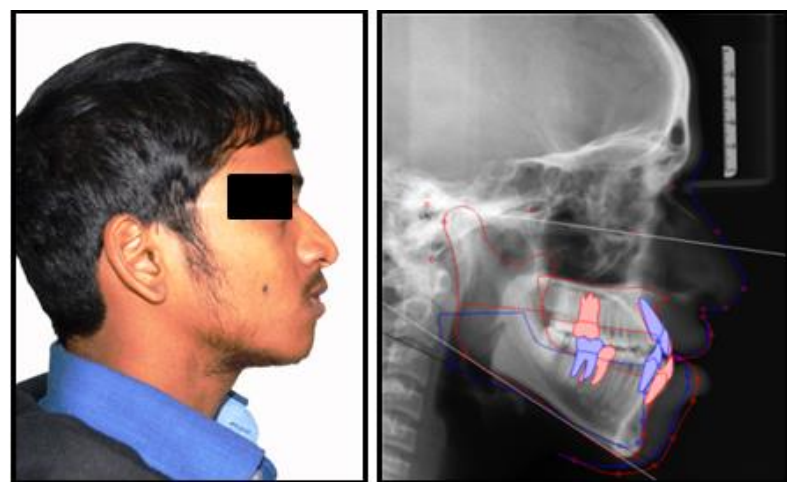

Fig. 3: Software Predicted Image \& Planned Cephalogram

1. Cephalometric radiographs of skeletal class I bimaxillary protrusion patients
Alterations: maxillary displacement at point $\mathrm{A}$ and mandibular displacement at point B.

Manipulations: maxillary segmental osteotomy (setback) and mandibular segmental osteotomy(set back)

There after the 20 original images of skeletal class I bimaxillary patients together with 20 predicted images were assessed.

2. Cephalometric radiographs of skeletal class II patients

Alterations: mandibular displacement at point B.

Manipulations: mandibular advancement (BSSO), and genioplasty

There after the 20 original images of skeletal class II patients together with 20 predicted images were assessed

3. Cephalometric radiographs of skeletal class III patients

Alterations: mandibular displacement at point B.

Manipulations: Mandibular setback (BSSO), maxillary segmental osteotomy (advancement)and genioplasty (setback).

There after the 10 original images of skeletal class III patients together with 10 predicted images were assessed. A total of 50 predicted pictures of patients were assessed comparing to original profile images.

Three rater groups were used to evaluate the facial attractiveness of the profile images including 50 Laypersons, 50 Orthodontists, 50 Dental students. All the raters were assessed regarding their reliability to evaluate the image attractiveness and diagnosed to be eligible for the study.The assessments are done using visual analogue scale(VAS) with 0 representing least attractiveness and 5 representing the most attractiveness according to the rater. The raters were given the $\mathrm{CD}$ (soft copy ) of profile images and questionnaire forms. The raters judged the profile attractiveness in distinct hours of the day to eliminate the influence of factors like tiredness on their point of view further more they were asked to observe the images in an over all view and make the decisions later (fast show technique) the questionnaires were completed and underwent statistical evaluations.

Table 1: Mean and central tendencies of predicted profile images of class I bimax patients according to raters group.

\begin{tabular}{|c|c|c|c|c|c|}
\hline Profiles & Judges & Number & Mean score & Standard deviation & Standard Error \\
\hline Class I BIMAX & Layman & 50 & 4.27 & 0.45 & 0.14 \\
\hline & Students & 50 & 3.74 & 0.54 & 0.17 \\
\hline & Orthodontists & 50 & 3.46 & 0.53 & 0.17 \\
\hline
\end{tabular}


Table 2: Mean and central tendencies of predicted profile images of class II patients according to raters group.

\begin{tabular}{|c|c|c|c|c|c|}
\hline Profiles & Judges & Number & $\begin{array}{c}\text { Mean } \\
\text { Score }\end{array}$ & $\begin{array}{c}\text { Standard } \\
\text { deviation }\end{array}$ & Standard Error \\
\hline Class II & Layman & 50 & 3.91 & 0.50 & 0.15 \\
\hline & Students & 50 & 3.51 & 0.72 & 0.22 \\
\hline & Orthodontists & 50 & 3.41 & 0.76 & 0.24 \\
\hline
\end{tabular}

Table 3: Mean and central tendencies of predicted profile images of class III patients according to raters group.

\begin{tabular}{|l|c|c|c|c|c|}
\hline Profiles & Judges & Number & Mean Score & $\begin{array}{c}\text { Standard } \\
\text { deviation }\end{array}$ & Standard Error \\
\hline Class III & Layman & 50 & 4.12 & 0.44 & 0.19 \\
\hline & Students & 50 & 3.63 & 0.50 & 0.22 \\
\hline & Orthodontists & 50 & 3.40 & 0.66 & 0.25 \\
\hline
\end{tabular}

Table 4: The central tendency indices of the predicted profile images of all patients according to all raters group

\begin{tabular}{|c|c|c|c|}
\hline Profile & Mean & Standard deviation & Ranking \\
\hline Class III & 3.71 & 0.36 & 2 \\
\hline Class II & 3.63 & 0.25 & 3 \\
\hline Class I BIMAX & 3.82 & 0.41 & 1 \\
\hline
\end{tabular}

\section{Results}

Predicted profile images of all the patients were attractive comparing to their original profile images irrespective of the malocclusion, highest scores regarding attractiveness were given to the predicted images of class I Bimax patients and the lowest scores were given to the predicted images of skeletal class II.

Predicted profile images of class I Bimax were rated attractive with a mean VAS(Visual Analogue Scale) score of 3.82 out of 5. Predicted profile images of class II were rated attractive with a mean VAS(Visual Analogue Scale) score of 3.54 out of 5. Predicted profile images of class III were rated attractive with a mean VAS(visual analogue scale) score of 3.71 out of 5 .

\section{Discussion}

The study suggested that the predicted profile images of all the patients were attractive and rankings were decreasing from class I bimaxillary class III and then class II. The least score of attractiveness were given to class II patients. The predicted profile images of class I bimaxillary patients were more attractive. ${ }^{3}$

Dental specialists (Orthodontists and Dental students) are more critical at their ratings than laypersons because of their specialized education, training background and scientific knowledge regarding dentofacial deformities; although, some differences were noted between them. ${ }^{4}$ Dentists are able to discriminate profile changes more accurately due to the knowledge received to detect severe deviations from the normal values. This finding may justify the observed slight differences between the laypersons and Orthodontists in the ranking of class III profile images. ${ }^{5}$

The rater's social class was more important in ranking of dental and skeletal beauty. Raters with a lower socio-economic situation are used to give fewer attractiveness scores than those with higher status. Different factors are influential on the ranking of the facial esthetics. ${ }^{6}$ As different assessments of the facial attractiveness were expressed by laypersons in some cases, Orthodontist must be aware of these ideas. ${ }^{7}$

By this, they will be able to cooperate with the patients and produce more similar beauty judgements increasing the success of treatments and achieving patients satisfaction in turn.

\section{Conclusion}

The results of the this study showed that the predicted profile images were attractive as suggested by three raters with class I bimaxillary most attractive and class II least attractive. In overall, laypersons and dental students ratings were somehow similar in the assessment of facial profile attractiveness in the predicted profile images.

\section{Source of Funding}

None.

\section{Conflict of Interest}

None. 


\section{References}

1. Riggio RE, Widaman KF, Tucker JS, Salinas C. Beauty is more than skin deep: components of attractiveness. Basic Appl Psychol 1991;12(4):423-9.

2. Vincent JA. A comparison of providers and consumers perception of facial profile attractiveness. Am J Orthod Dentofac Orthop 1998;114:631-7.

3. Orsini MG, Huang GJ, Kiyak HA, Ramsay DS, Bollen $\mathrm{AM}$, Anderson NK et al, Methods to evaluate profile preferences for the antero-posterior position of the mandible. Am J Orthod Dentofacial Orthop 2006;130(3):283-91.

4. Giddon DB. Orthodontic applications of psychological and perceptual studies of facial esthetics. Semin Orthod 1995;1(2):82-93.

5. Konstiantos KA, O'Reilly MT, Close J. The validity of the prediction of tissue profile changes after LeFort I osteotomy using the dentofacial planner. Am J Orthod Dentofacial Orthop 1994;105(3):241-9. 